\title{
Erosion at the inner wall of JET during the discharge campaigns 2001-2009
}

\author{
M. Mayer ${ }^{\mathrm{a}, *}$, S. Krat ${ }^{\mathrm{a}, \mathrm{b}}$, J.P. Coad ${ }^{\mathrm{c}}$, A. Hakola ${ }^{\mathrm{d}}$, J. Likonen ${ }^{\mathrm{d}}$, S. Lindig ${ }^{\mathrm{a}}$, A. Widdowson ${ }^{\mathrm{c}}$, and \\ JET-EFDA contributors ${ }^{+}$ \\ JET-EFDA, Culham Science Centre, Abingdon, OX14 3DB, UK \\ ${ }^{a}$ Max-Planck-Institut für Plasmaphysik, EURATOM Association, Boltzmannstr. 2, D-85748 Garching, \\ Germany. \\ ${ }^{b}$ National Research Nuclear University “MEPhI”, Moscow Kashirskoe road 31, 115409, Russia. \\ ${ }^{c}$ Culham Science Centre, EURATOM/UKAEA - Fusion Association, Abingdon, Oxfordshire OX14 \\ $3 D B, U K$. \\ ${ }^{d}$ VTT, Association EURATOM-Tekes, PO Box 1000, FI-02044 VTT, Finland.
}

\begin{abstract}
The erosion of $\mathrm{Be}, \mathrm{C}, \mathrm{Ni}$ and $\mathrm{W}$ marker layers was investigated with long-term samples exposed during the discharge periods 2001-2004 and 2005-2009. The markers were mounted in carbon wall tiles between the inner wall guard limiters (IWGLs) and at positions between the lower end of the IWGLs and the inner divertor entrance. They were analyzed using ion beam analysis techniques before and after exposure. All samples showed strong erosion of all elements. The erosion is predominantly due to neutral particles created by charge-exchange (CX) collisions. The erosion of carbon was about $8 \times 10^{19}$ atoms $/ \mathrm{cm}^{2}$ (about $8 \mu \mathrm{m}$ ) during the 2005-2009 campaign, which gives a total erosion of carbon from the recessed areas between the IWGLs of about $230 \mathrm{~g}$ : The inner wall is therefore an important net carbon source. The erosion of the four different elements allows a rough reconstruction of the energy spectrum of the eroding CX neutrals.
\end{abstract}

\section{PACS}

52.40Hf, 52.55.Fa, 82.80.Yc

PSI-20 keywords 
Charge exchange; Erosion \& Deposition; First wall; Impurity sources; JET

Corresponding author address

Max-Planck-Institut für Plasmaphysik, EURATOM Association, Boltzmannstr. 2, D85748 Garching, Germany

\section{Corresponding Author e-mail}

matej.mayer@ipp.mpg.de

\section{Presenting Author}

Matej Mayer

Presenting Author e-mail

matej.mayer@ipp.mpg.de 


\section{Introduction}

Erosion and redeposition of plasma facing materials are important processes for material lifetime and tritium inventory. The inner wall of the JET vessel consists of inner wall guard limiters (IWGLs) with recessed areas between the limiters. As has been already observed previously, these recessed areas show a substantial net material erosion

$[1,2,3]$. Based on spectroscopic investigations of the inner wall it has been already argued that a large fraction of the carbon redeposited in the inner divertor may originate from here [4].

Because these areas are recessed by several centimeters behind the IWGLs and should not receive large particle fluxes, it is not obvious how such a large erosion can be achieved. It was already assumed in [1], that mainly charge-exchange (CX) neutral particles are responsible for the observed erosion. In contrast to these findings at JET, it was shown at ASDEX Upgrade (AUG) that mainly ion fluxes are responsible for erosion at the AUG inner heat shield [5]. Although the geometries of the inner walls of JET and AUG are not fully comparable (at AUG the whole inner heat shield operates as limiter, while JET has IWGLs and recessed areas in between), this rose the question if ion fluxes may also play a role at the inner wall of JET. In addition to the question about the nature of the eroding flux, information about the energy distribution of the eroding species is highly desirable. The inner wall is generally difficult to diagnose, but the erosion of different materials with varying sputtering thresholds allows to achieve at least some information about this energy spectrum.

This paper describes experimental results from long-term samples during the 2001-2004 and 2005-2009 operational campaigns at the recessed parts of the JET inner wall between the IWGLs. The erosion/deposition pattern at the IWGLs is described elsewhere [6,7]. 


\section{Experimental}

Nine long-term samples (LTS) were exposed during the 2001-2004 and 2005-2009 discharge campaigns. The samples were made from fine-grain graphite and flash-mounted as sachet inserts in carbon protection tiles between the IWGLs. Four samples were mounted in octant 4 at different poloidal locations, six samples were mounted close to the inner midplane in different octants.

One third of the surface of the LTS exposed in 2001-2004 was coated with a thin tungsten layer having an initial thickness of about $50 \mathrm{~nm}$ using physical vapor deposition. About $2 \mu \mathrm{m}$ thick nickel layers were deposited on another third of the surface, but delaminated from the substrate during exposure at JET. The remaining third was left blank in order to measure deposition of heavier elements, especially Inconel constituents. Half of the surface area of the LTS exposed in 2005-2009 were coated with about $50 \mathrm{~nm}$ tungsten and 8-10 $\mu \mathrm{m}$ carbon on top: The $\mathrm{W}$ layer served as a marker for the interface between the carbon substrate and the carbon marker layer. The second half of the LTS contained $5 \mu \mathrm{m}$ beryllium layers on copper substrate. The Be/Cu samples were clamped in a stainless steel housing.

Samples with a magnetically operated flap were mounted in octants 3 and 5 . The flap was only open during plasma discharges and closed during wall conditioning, such as helium glow discharge cleaning or beryllium evaporations. Four different samples were placed behind the flap: $50 \mathrm{~nm} \mathrm{~W}$ on silicon substrate; $2.5 \mu \mathrm{m}$ nickel on silicon substrate with gold interlayer; $2.5 \mu \mathrm{m}$ beryllium on copper substrate; and $9 \mu \mathrm{m}$ (2001-2004) or $10 \mu \mathrm{m}$ (20052009) carbon. In 2001-2004 a carbon foil was used, and carbon layers with tungsten interlayer on fine grain graphite substrate in 2005-2009. 
The samples were analyzed using ion beam analysis methods before and after exposure. Rutherford backscattering (RBS) using $1.5 \mathrm{MeV}^{4} \mathrm{He}$ was used for measuring the thicknesses of the $\mathrm{W}$ layers. 1.5 MeV protons were used for $\mathrm{Ni}$ and Be layers, and 1.5 and $3 \mathrm{MeV}$ protons for C layers. A silicon solid state detector at a scattering angle of $165^{\circ}$ with a solid angle of 1.16 msr was used. The program SIMNRA [8] with SRIM 2010 stopping powers [9] and non-Rutherford scattering cross-sections from [10] was used for quantitative evaluation of the RBS spectra.

\section{Results and discussion}

The poloidal and toroidal distributions of the erosion of Be, $\mathrm{C}$ and $\mathrm{W}$ are shown in Fig. 1. The erosions of all three elements are almost homogeneous in both directions. Deposition of other elements, for example of Be due to wall conditioning or re-deposition of eroded elements (especially nickel from the Inconel wall) was not observed. The recessed part of the inner wall between the inner wall guard limiters has a total area of about $15 \mathrm{~m}^{2}$. With the measured carbon erosion of about $8 \mu \mathrm{m}$ in 2005-2009 this gives a total carbon erosion of about $230 \mathrm{~g}$ during that time. As has been shown in [7] this is an important contribution to the total carbon balance. The erosion of beryllium is smaller by a factor of about two: If the inner wall would have been made from beryllium rather than from carbon, then the erosion would have been about $90 \mathrm{~g}$. It is also noteworthy that the erosion of tungsten is only about $2 \%$ of the carbon erosion.

After exposure during the 2001-2004 campaign the initial thickness of about $50 \mathrm{~nm} \mathrm{~W}$ on fine-grain graphite has decreased to about $25 \mathrm{~nm}$, see Fig. 1. The RBS spectra indicated, that the remaining $\mathrm{W}$ layer still covered the whole surface. This was confirmed by secondary 
electron microscopy (SEM) images, see Fig. 2: The top image shows the surface topography with large pores characteristic for fine-grain graphite, while the bottom image recorded with backscattered electrons is dominated by material contrast: Tungsten appears bright, and carbon appears dark. The observed grayish image without strong brightness variations indicates a relatively homogeneous $\mathrm{W}$ distribution. SEM images of the Be layers exposed in 2005-2009 showed a very similar picture. Erosion of rough surfaces by ions is typically characterised by a very inhomogeneous erosion, with a large erosion on plasma-inclined faces and a small erosion or even deposition on shadowed faces of the rough surface $[11,12,13]$. The rather homogeneous erosion on the samples therefore indicates that the contribution of ions to the observed erosion is small, and the erosion is mainly due to chargeexchange neutral particles. This is not too astonishing, because the samples are recessed behind the IWGLs and are not struck by magnetic field lines, see for example the schematics of the magnetic X-point configuration in [14]. Nevertheless, it is noteworthy that the observed homogeneous erosion pattern in these recessed areas is very different from the strongly inhomogeneous pattern in areas hit by large ion fluxes [13].

The erosion of the materials on the flap samples is shown in Fig. 3 for the 2001-2004 and 2005-2009 campaigns. The average values for the samples in octant 3 and 5 are shown. The different materials have very different threshold energies for sputtering by D: For W the threshold energy is $201 \mathrm{eV}$ [15], for Ni $30.8 \mathrm{eV}$ [15], for Be $9.8 \mathrm{eV}$ [15], and only 1-2 eV for the chemical sputtering of $C[16,17]$. The observed erosion of the different materials therefore contains information about the energy spectrum of the incident flux of CX-neutrals, which cannot be measured directly at the inner wall by other methods.

The total erosion $S$ of one element during the exposure is given by 


$$
S=\int Y(E) \Gamma(E) d E
$$

with $Y(E)$ the energy-dependent sputtering yield and $\Gamma(E)$ the differential flux integrated over the whole campaign. The fit formulas for the experimental physical sputtering yields at normal incidence from [15] have been used for $\mathrm{Be}, \mathrm{Ni}$ and $\mathrm{W}$, and the chemical erosion yield for carbon at $200^{\circ} \mathrm{C}$ (which is about the wall temperature during the campaign) from [16,17]. The mean energy $E_{0}$ of the eroding flux is given by

$$
E_{0}=\frac{1}{S} \int E Y(E) \Gamma(E) d E
$$

For the energy distribution of the CX-flux $\Gamma(E)$ we assume a functional form according to

$$
\Gamma(E)=A_{1} e^{-E / \lambda_{1}}+A_{2} e^{-E / \lambda_{2}},
$$

i.e. a superposition of two exponential decays with decay lengths $\lambda_{1}$ and $\lambda_{2}$. This shape of the CX energy spectrum is motivated by the shape of measured CX-spectra at the low-field side of ASDEX Upgrade [18], see Fig. 4. The campaign-integrated energy spectra for the 2001-2004 and 2005-2009 campaigns are shown in Fig. 4, the calculated erosion yields from these fluxes are compared to the experimental data in Fig. 3. The large difference of the measured $\mathrm{C}$ and Be erosion can be only explained by a very strong increase of the flux towards low energies, as shown in Fig. 4. These low-energetic particles are still able to erode carbon by chemical erosion due to the very low threshold energy of chemical sputtering, but their energy is too low for physical sputtering of other elements. Ni and $\mathrm{W}$ are eroded by particles with a very broad energy spectrum, so that only information about the total number of higher-energetic particles, but no detailed information about their energy spectrum at energies above about $300 \mathrm{eV}$ can be obtained. This has been taken into account by assuming 
a constant energy spectrum above $300 \mathrm{eV}$, i.e. by assuming $\lambda_{2}=. \infty$. As mentioned above, the sputtering yields at normal incidence were used. As has been shown at ASDEX Upgrade, the incident CX-flux has also an angular distribution [18]. Information about the angular distribution cannot be obtained by the described method.

The erosion of Be and $\mathrm{C}$ on the flap samples is characterised by a large ratio of carbon to beryllium erosion of about ten, see Fig. 3. This ratio decreases at the recessed inner-wall areas to about 2, see Fig. 1: This means qualitatively, that the contribution of higher-energetic particles increases at the recessed areas between the IWGLs. A more quantitative statement, however, is difficult because the reconstruction of the CX-flux gets very crude if based on only two materials.

JET plasmas are typically ramped up and down as limiter plasmas at the IWGLs. The ratio of limiter phase time to X-point time is roughly $1: 3$, with $50-60 \%$ of the limiter plasma time in contact with the central part of the limiter [19]. It can be argued, that much of the observed erosion happens during these phases. With current knowledge it is difficult to distinguish erosion during the limiter phases from erosion during the $\mathrm{X}$-point phases. However, the rather homogeneous erosion of the LTS in poloidal direction is an indication, that erosion during the limiter phases may be not the dominant process, as has been already discussed for the observed erosion pattern at the IWGLs [19].

\section{Conclusions}

The erosion of $\mathrm{Be}, \mathrm{C}, \mathrm{Ni}$ and $\mathrm{W}$ marker layers was investigated with long-term samples (LTS) exposed during the discharge periods 2001-2004 and 2005-2009. The markers were analyzed using ion beam analysis techniques before and after exposure and were mounted in carbon wall tiles between the IWGLs and at positions between the lower end of the IWGLs 
and the inner divertor entrance.

All samples showed strong erosion of the investigated markers. The erosion is predominantly due to neutral particles created by charge-exchange (CX) collisions. The erosion was almost constant in toroidal and poloidal directions over the whole inner wall.

The erosion of the four different elements allows a rough reconstruction of the energy spectrum of the eroding CX neutrals. The spectrum is characterized by a very steep increase of the flux of low energetic deuterium atoms with energies below about $200 \mathrm{eV}$, the main erosion mechanism of carbon is therefore chemical erosion. The total erosion of carbon during the 2005-2009 campaign from the recessed areas was about $230 \mathrm{~g}$. In addition strong carbon erosion/redeposition was observed at the IWGLs [6,7]. The inner wall was therefore an important, but not the only, net carbon source. The erosion of beryllium with the ITERlike wall is expected to be smaller by a factor of about two due to the absence of chemical erosion.

\section{Acknowledgements}

This work was partly supported by the Russian Ministry of Education and Science and ROSATOM contracts. This work was supported by EURATOM and carried out within the framework of the European Fusion Development Agreement. The views and opinions expressed herein do not necessarily reflect those of the European Commission. 

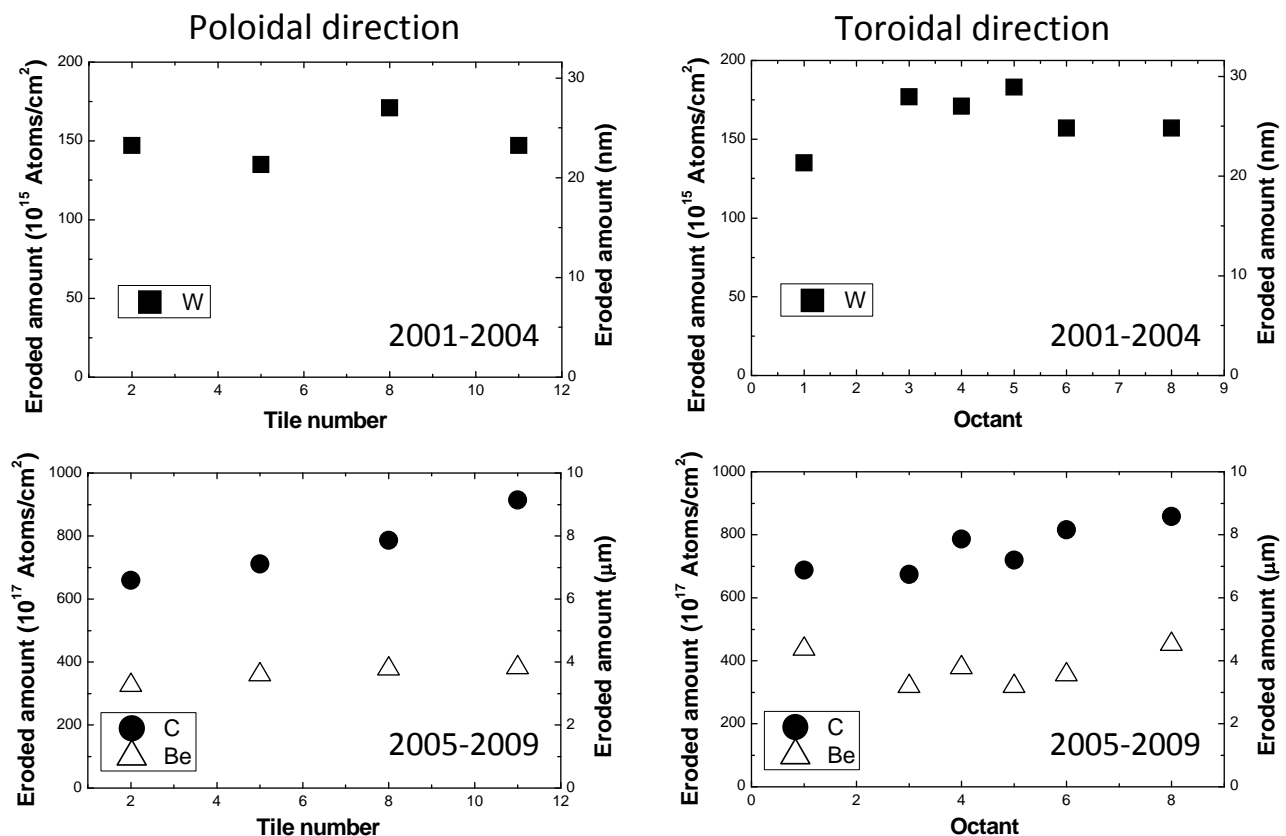

Fig. 1: Poloidal and toroidal distributions of the erosion of W, measured during the 20012004 discharge campaign, and the erosion of Be and C measured during the 2005-2009 discharge campaign on sachet inserts at the inner wall between the inner wall guard limiters. The measured data are in atoms $/ \mathrm{cm}^{2}$, the right-hand scales are only given for convenience. The theoretical density of $\mathrm{W}$ and a density of $10^{23}$ atoms $/ \mathrm{cm}^{3}$ was used for Be and C. 


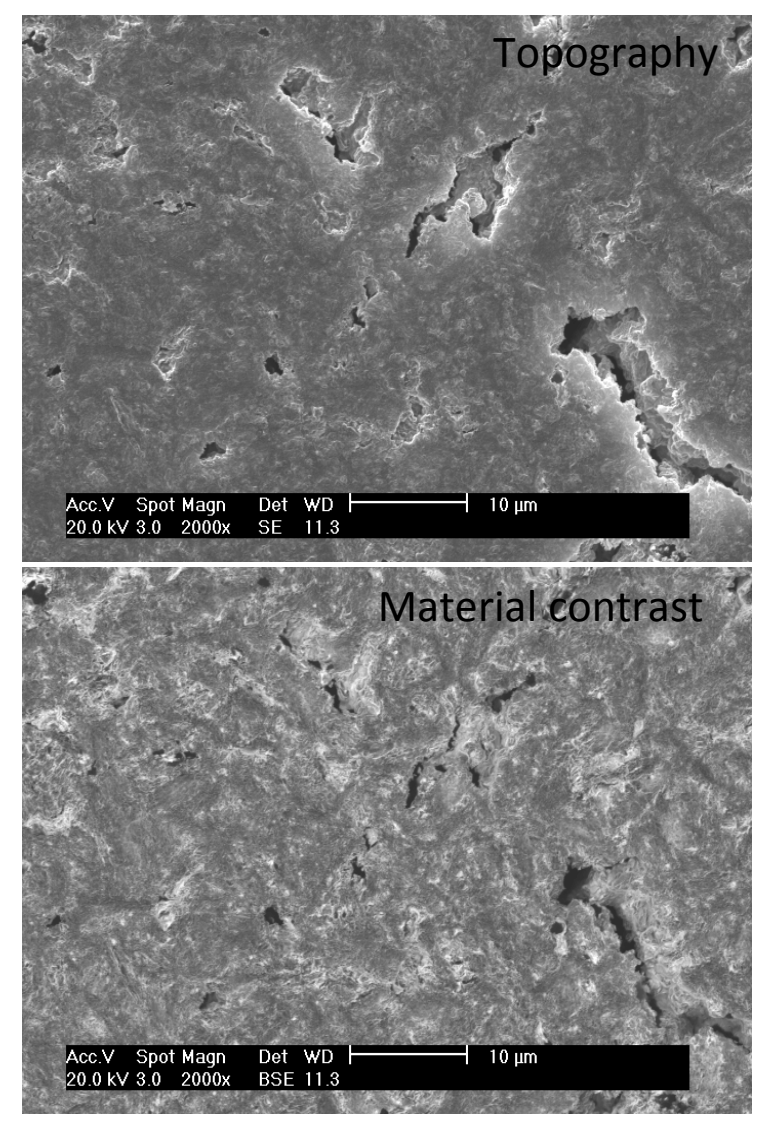

Fig. 2: Secondary electron microscopy images using secondary electrons (top) and backscattered electrons (bottom) of the W layer on fine-grain graphite exposed 2001-2004. 

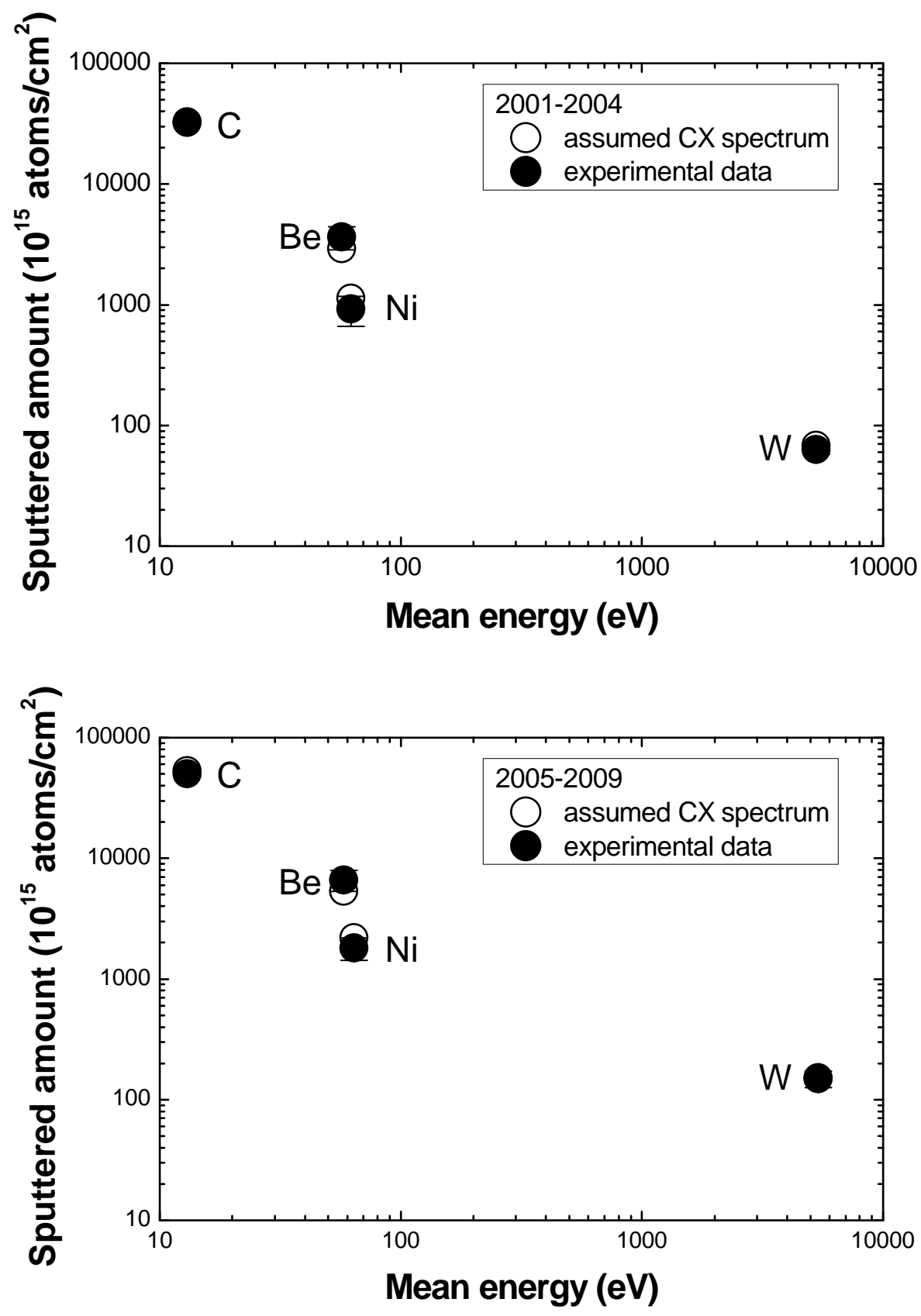

Fig. 3: Erosion of Be, C, Ni and W determined on the flap samples during the 2001-2004 campaign (top) and during the 2005-2009 campaign (bottom) as a function of the mean energy of the eroding CX-flux (see text). Red dots: Experimental data including error bars; black dots: Calculated erosion using the assumed CX-spectra shown in Fig. 4. 


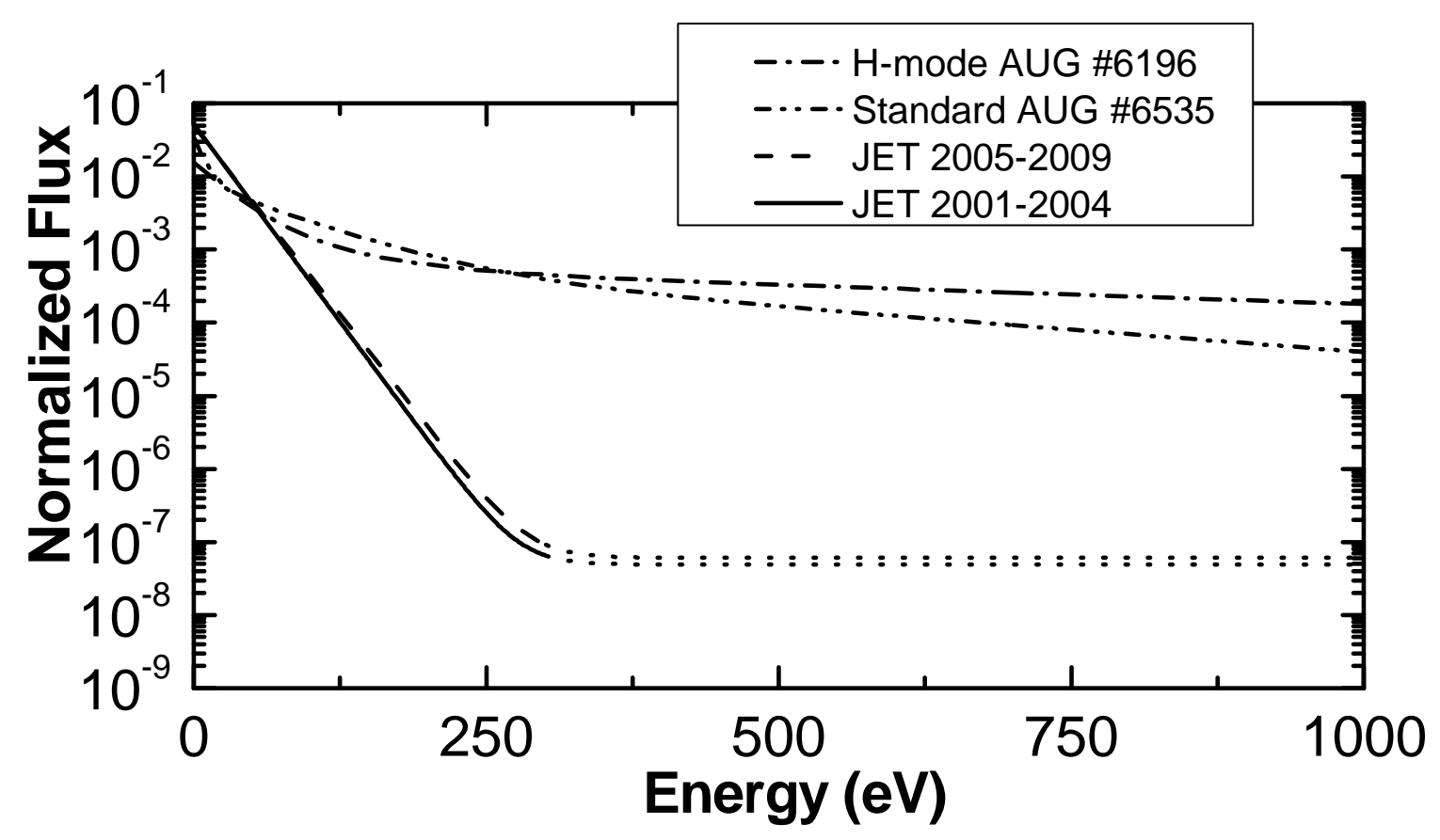

Fig. 4: Reconstructed CX-spectra from the measured erosion of $\mathrm{Be}, \mathrm{C}, \mathrm{Ni}$ and $\mathrm{W}$ determined on the flap samples during the 2001-2004 and 2005-2009 campaigns. Measured CX spectra at ASDEX Upgrade [18] are shown for comparison. All spectra are normalized to the same area.

1 M. Mayer, R. Behrisch, P. Andrew, A.T. Peacock, J. Nucl. Mater. 241 (1997) 469 2 M. Mayer, R. Behrisch, P. Andrew, J.P. Coad, and A.T. Peacock, Physica Scripta T81 (1999) 13

3 M. Mayer, R. Behrisch, K. Plamann, P. Andrew, J.P. Coad, and A.T. Peacock, J. Nucl. Mater. 266 (1999) 604

4 G.F. Matthews, M. Stamp, P. Coad, J. Likonen, V. Philipps, M. Rubel, J.D. Strachan, and JET EFDA Contributors, Europhysics Conference Abstracts 27A (2003) P-3.198

5 K. Krieger, A. Geier, X. Gong, H. Maier, R. Neu, V. Rohde, and ASDEX Upgrade Team, J. Nucl. Mater. 313 (2003) 327

$6 \mathrm{~S}$. Krat et al., this conference, P2-30 
7 A. Widdowson et al., this conference, P2-60

8 M. Mayer, SIMNRA User's Guide, Report IPP 9/113, Max-Planck-Institut für Plasmaphysik, Garching, Germany, 1997

9 J.F. Ziegler, M.D. Ziegler, and J.P. Biersack, Nucl. Instr. Meth. B 268 (2010) 1818 10 A.F. Gurbich, Nucl. Instr. Meth. B 136-138 (1998) 60

11 M. Mayer, J. Likonen, J.P. Coad, H. Maier, M. Balden, S. Lindig, E. Vainonen-Ahlgren, V. Philipps, and JET-EFDA Contributors, J. Nucl. Mater. 363-365 (2007) 101-106 12 M. Mayer, M. Andrzejczuk, R. Dux, E. Fortuna-Zalesna, A. Hakola, S. Koivuranta, K.J. Kurzydlowski, K. Krieger, J. Likonen, G. Matern, R. Neu, G. Ramos, M. Rasinski, V. Rohde, K. Sugiyama, A. Wiltner, W. Zielinski, and ASDEX-Upgrade team, Phys. Scr. T138 (2009) 014039

13 K. Schmid, M. Mayer, C. Adelhelm, M. Balden, S. Lindig and the ASDEX Upgrade team, Nuclear Fusion 50 (2010) 105004

14 J.D. Strachan, G. Corrigan, M. Stamp, J. Spence, J. Zacks, and JET-EFDA Contributors, J. Nucl. Mater. 390-391 (2009) 92

15 W. Eckstein, C. García-Rosales, J. Roth, and W. Ottenberger, Sputtering Data, Report IPP 9/82, Max-Planck-Institut für Plasmaphysik, Garching, Germany, 1993

16 J. Roth and C. García-Rosales, Nuclear Fusion 36 (1996) 1647

17 J. Roth and C. García-Rosales, Nuclear Fusion 37 (1997) 897

18 H. Verbeek, J. Stober; D.P. Coster; W. Eckstein; and R. Schneider, Nuclear Fusion 38 (1998) 1789

19 J P Coad, S Gruenhagen, D E Hole, A Hakola, S Koivuranta, J Likonen, M Rubel, A

Widdowson and JET-EFDA contributors, Physica Scripta T145 (2011) 014003 